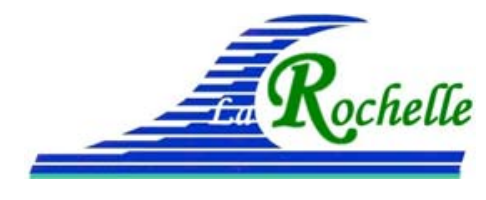

$X V^{\text {èmes }}$ Journées Nationales Génie Côtier - Génie Civil

La Rochelle, 29 au 31 mai 2018

DOI:10.5150/jngcgc.2018.010 (C) Editions Paralia CFL

disponible en ligne - http://www.paralia.fr - available online

\title{
Apport de la télédétection spatiale à la représentation des champs de vents cycloniques
}

\author{
Yann KRIEN ${ }^{1}$, Gaël ARNAUD ${ }^{1}$, Raphaël CECE ${ }^{1}$, Jamal KHAN ${ }^{2,3}$, \\ Ali BEL MADANI ${ }^{4}$, Didier BERNARD ${ }^{1}$, A.K.M.S. Islam ${ }^{3}$, Fabien DURAND ${ }^{2}$, \\ Laurent TESTUT ${ }^{5}$, Philippe PALANY ${ }^{4}$, Narcisse ZAHIBO ${ }^{1}$
}

1. Université des Antilles, Laboratoire de Recherche en Géosciences et Energies (LARGE), Fouillole, Pointe-à-Pitre 97157, Guadeloupe, France.

2. Laboratoire d'Etudes en Géophysique et Océanographie Spatiales (LEGOS), 14 Avenue Edouard Belin, Toulouse, France.

3. IWFM, BUET, Dhaka, Bangladesh.

4. Météo France, DIRAG, Martinique, France.

5. Université de La Rochelle, LIENSs, UMR 7266 CNRS, La Rochelle, France.

Contact:ykrien@gmail.com

\section{Résumé :}

La reproduction des champs de vent cycloniques est une étape cruciale pour l'étude des impacts des cyclones en termes de submersion marine, de transport sédimentaire, ou encore d'états de mer. Qu'il s'agisse de prévision ou de caractérisation de l'aléa, cette phase repose à ce jour encore très largement sur des modèles paramétriques dont la validité n'est établie qu'à l'aide d'un nombre limité d'observations. Par ailleurs, le choix d'un modèle plutôt qu'un autre relève bien souvent de l'arbitraire plutôt que de considérations scientifiques objectives. Ce travail a pour principal objectif de montrer que la télédétection spatiale permet aujourd'hui de mieux représenter la structure des champs de vent cycloniques, et donc de choisir le modèle qui se rapproche le plus de la réalité en fonction du cas d'étude. Les résultats obtenus suggèrent par ailleurs que la plupart des modèles paramétriques utilisés à ce jour présentent des biais systématiques. Le travail initié dans cet article devrait permettre à la communauté scientifique d'améliorer la représentation des champs de vent cycloniques et de mieux évaluer les impacts de ces événements extrêmes.

\section{Mots-clés :}

Télédétection spatiale, Cyclones, Ouragans, Vents paramétriques, Submersions marines, Vagues, CYGNSS, ASCAT.

\section{Introduction}

Les champs de vent cycloniques sont encore largement représentés dans la communauté scientifique à l'aide de modèles paramétriques. Qu'il s'agisse de laboratoires de recherche, de bureaux d'étude, de compagnies de réassurance, ou même de centres d'alertes, les études d'impacts cycloniques font encore très souvent appel à ces 


\section{Thème 1 - Hydrodynamique côtière}

approches qui consistent à reconstruire les champs de vent et de pression en fonction du temps à l'aide d'un nombre réduit de paramètres d'entrée, comme la localisation du cyclone, la vitesse de vent maximale, la pression au centre, ou encore les rayons de vent à 64,50 , et 34 nœuds. Le choix du modèle a des conséquences importantes sur la capacité de l'étude à estimer correctement ou non les impacts cycloniques, en termes de d'états de mer ou encore de submersions marines (LIN \& CHAVAS, 2012). Il s'agit donc d'une étape cruciale pour la prévision opérationnelle ou encore la caractérisation des aléas. Pourtant, ce choix repose rarement sur des critères très objectifs. A titre d'exemple, le modèle de HOLLAND (1980) est toujours très utilisé, bien que l'on sache aujourd'hui qu'il présente de réels défauts (WILLOUGHBY \& RAHN, 2004).

La télédétection spatiale a fait d'énormes progrès ces dernières années. De nombreux satellites ont été déployés afin d'apporter des informations sur la structure des vents cycloniques. Les méthodes développées à cet effet (imagerie infrarouge, diffusiométrie, radiométrie, réflectométrie GNSS) ont d'ors et déjà prouvé leur efficacité (MORRIS \& RUF, 2017), et sont de plus en plus utilisées conjointement avec les mesures in-situ (ballons sondes, avions de chasse de la NOAA, etc) pour les prévisions cycloniques dans les centres d'alerte. C'est donc assez naturellement qu'on en vient à se demander si ces données ne pourraient pas être aussi utilisées pour améliorer les modèles de vent paramétriques, et donc les résultats des études d'aléas cycloniques.

Dans cette étude, nous nous intéresserons plus particulièrement à la mission CYGNSS de la NASA, spécifiquement conçue pour estimer les vents de surface en condition cyclonique, avec une couverture spatiale et temporelle inégalée (plusieurs passages par jour au dessus d'une même zone dans la bande intertropicale, RUF et al., 2016).

\section{Données et méthodes}

\subsection{Sélection des événements cycloniques}

Avec 6 ouragans majeurs, dont deux de catégorie 5, la saison cyclonique 2017 a été particulièrement active en Atlantique. Ces événements ont fait par ailleurs l'objet d'un grand nombre de mesures in-situ ou de télédétection. Ils constituent donc une bonne base de données pour les besoins de notre étude. Nous avons par conséquent considéré l'ensemble des ouragans de la saison 2017 en Atlantique, ainsi qu'un certain nombre de cyclones dans le Pacifique Est. En tout, cela représente 16 évènements: Dora, Eugene, Franklin, Gert, Harvey, Hilary, Irma, Irwin, Jose, Katia, Kenneth, Lee, Maria, Max, Norma, et Otis. Pour chacun de ces ouragans, nous avons collecté les informations données par le NHC (National Hurricane Center) toutes les 6h: localisation du cyclone, vitesse de vent maximale, et rayons correspondants aux vents à 34, 50, et 64 nœuds dans chacun des quadrants. 


\section{XVèmes Journées Nationales Génie Côtier - Génie Civil \\ La Rochelle, 29 au 31 mai 2018}

\subsection{Données de télédétection spatiale}

Les données de la mission CYGNSS (RUF et al., 2016) ont été recueillies pour l'ensemble de la saison 2017. Cette mission consiste en une constellation de 8 microsatellites qui estiment les vitesses de vent à la surface de l'océan par réflectométrie GNSS (MORRIS \& RUF, 2017 ; RUF et al., 2016). Cette approche consiste à comparer les signaux radar émis directement par les stations GNSS avec ceux réfléchis par la surface. La bande de fréquence utilisée (bande L) permet de s'affranchir des problèmes liés aux fortes précipitations associées aux cyclones. Par ailleurs, la présence de plusieurs satellites permet d'avoir une bonne couverture temporelle (plusieurs passages par jour au dessus d'une même zone). Les données considérées ici (v2.0, produit nbrc, Niveau 2) ont une résolution spatiale d'environ $25 \mathrm{~km}$. Les validations faites sur la saison 2017 indiquent des erreurs quadratiques moyennes de l'ordre de 17\% pour des vents supérieurs à $20 \mathrm{~m} / \mathrm{s}$ (RUF et al., 2018), dont la moitié associée à des biais.

A titre de comparaison, nous avons aussi collecté les données acquises par les diffusiomètres ASCAT (FIGA-SALDANA et al., 2002) montés sur les satellites MetOp-A (2006) et MetOp-B (2012), avec une résolution équivalente (environ $25 \mathrm{~km}$ ). Notons d'ors et déjà que cette résolution $(25 \mathrm{~km})$ est du même ordre de grandeur que le rayon pour lequel le vent est maximum (au niveau du mur de l'oeil). Ces données ne permettent donc pas de représenter la région de vitesse maximale où les gradients sont importants. En revanche, le gradient de vitesse est suffisamment faible au delà de quelques dizaines de kilomètres pour que cette résolution y soit suffisante, a priori.

\subsection{Modèles de vent paramétriques}

Dans cet article, nous comparons les vitesses estimées par télédétection avec celles obtenues pour 4 formules de vents paramétriques (voir tableau 1). Pour chaque cyclone sélectionné, chaque formule, et chaque point de mesure CYGNSS/ASCAT, la vitesse de vent "paramétrique" en surface est calculée à partir des données collectées auprès du NHC (voir 2.1). Les principales étapes de ce calcul sont les suivantes :

a) La composante du champs de vitesse associée au déplacement du cyclone est estimée à chaque pas de temps suivant l'approche de LIN \& CHAVAS (2012).

b) Cette composante est retranchée des vitesses observées par le NHC.

c) Les vitesses en surface sont converties en vitesses en haut de la couche limite atmosphérique en les multipliant par un facteur $\mathrm{SWRF}=0.9$.

d) Les rayons de vents maximums sont calculés pour chaque quadrant à partir des informations dont on dispose sur les rayons de vents. On obtient jusqu'à 3 valeurs par quadrant: une à partir du rayon de vent à 64 nœuds $\left(R_{m 64}\right)$, une autre à partir du rayon de vent à 50 nœuds $\left(R_{m 50}\right)$, et enfin une à partir du rayon de vent à 34 nœuds $\left(R_{m 34}\right)$.

e) Les valeurs de $R_{m 64}, R_{m 50}$, et $R_{m 34}$ sont déterminées pour l'azimut correspondant au point de mesure ASCAT ou CYGNSS en effectuant une interpolation de type spline. 


\section{Thème 1 - Hydrodynamique côtière}

f) On calcule les valeurs de vitesses au point d'étude données par la formule empirique considérée (tableau 1). Il y a jusqu'à 3 valeurs, selon que l'on a calculé précédemment uniquement $R_{m 34}, R_{m 50}$ et $R_{m 34}$, ou tous les rayons de vent maximum.

g) La vitesse "paramétrique" au point d'étude est estimée à partir d'une méthode de pondération des vitesses établies à l'étape précédente (HU et al., 2012).

h) Cette vitesse correspond à une vitesse en haut de la couche limite atmosphérique. La vitesse correspondante en surface est estimée en la divisant par le facteur SWRF=0.9.

i) La vitesse obtenue à l'étape précédente est ajoutée à celle de l'étape a) pour avoir la vitesse finale "paramétrique", qui peut être ensuite comparée à celle observée par les missions CYGNSS et/ou ASCAT.

Tableau 1. Synthèse des formules paramétriques testées. $V_{m}$ et $R_{m}$ représentent respectivement la vitesse de vent maximale et le rayon de vent maximal. $r$ est la distance au centre du cyclone, et f le coefficient de Coriolis.

\begin{tabular}{|c|c|c|}
\hline Nom & Référence & Formule \\
\hline E11 & $\begin{array}{c}\text { EMANUEL \& } \\
\text { ROTUNNO (2011) }\end{array}$ & $V(r)=\frac{2 r\left(R_{m} V_{m}+0.5 f R_{m}^{2}\right)}{R_{m}^{2}+r^{2}}-\frac{f r}{2}$ \\
\hline E04 & EMANUEL (2004) & $\begin{array}{c}V(r)=V_{m} \frac{R_{0-r}}{R_{0}-R_{m}}\left(\frac{r}{R_{m}}\right)^{m}\left(\frac{(1+b)(n+m)}{n+m\left(\frac{r}{R_{m}}\right)^{2(n+m)}}\right. \\
\left.+\frac{b(1+2 m)}{1+2 m\left(\frac{r}{R_{m}}\right)^{2 m+1}}\right)^{0.5} \\
\text { avec } \mathrm{b}=0.25, \mathrm{~m}=1.6, \mathrm{n}=0.9, \mathrm{R}_{0}=420 \mathrm{~km}\end{array}$ \\
\hline H8O & HOLLAND (1980) & $\begin{array}{c}V(r)=\sqrt{\left(\frac{R_{m}}{r}\right)^{B} \frac{B \Delta P \exp \left(-\left(\frac{R_{m}}{r}\right)^{B}\right)}{\rho}+\frac{r^{2} f^{2}}{4}-\frac{f r}{2}} \\
\quad \text { avec }=\frac{V_{m}^{2} e \rho+f V_{m} R_{m} e \rho}{\Delta P}, \rho=1.15, e=\exp (1)\end{array}$ \\
\hline W06 & $\begin{array}{l}\text { WILLOUGHBY et al. } \\
\qquad \text { (2006) }\end{array}$ & $\begin{array}{c}\text { Pour } 0 \leq r \leq R_{m}: V(r)=V_{m}\left(\frac{r}{R_{m}}\right)^{n} \text { avec } n=0.79 \\
\text { Pour } r \geq R_{m}: V(r)=V_{m} \exp \left(-\frac{r-R_{m}}{X}\right) \text { avec } X=243 \mathrm{~km}\end{array}$ \\
\hline
\end{tabular}

\section{Résultats}

\subsection{Domaines de validité des données CYGNSS et ASCAT}

Dans un premier temps, les données CYGNSS et ASCAT sont comparées avec les vitesses "paramétriques" moyennées sur l'ensemble des formules. L'objectif est de voir si les biais sont ou non important, en fonction de l'intensité du cyclone ou de la distance du point considéré au centre $(r)$. Des biais faibles indiquent une cohérence entre les données de télédétection et les formules paramétriques, ce qui suggère que les résultats sont relativement fiables a priori. A l'inverse, des biais importants laissent à penser que les vitesses estimées par les formules paramétriques et/ou la télédétection posent 


\section{XVèmes Journées Nationales Génie Côtier - Génie Civil La Rochelle, 29 au 31 mai 2018}

problème. Les résultats sont présentés sur la figure 1. Ils révèlent des biais faibles pour les données ASCAT pour des rayons $r$ supérieurs aux rayons à 34 nœuds. A l'inverse, les biais sont de plus en plus négatifs lorsque les vitesses augmentent (rayon plus petit et augmentation de l'intensité du cyclone). Ces résultats sont cohérents avec ceux présentés par de nombreux auteurs (MORRIS \& RUF, 2017): les diffusiomètres ASCAT estiment correctement les vents faibles ou modérés, mais sous-estiment très largement les vents extrêmes. Les biais obtenus avec les données CYGNSS semblent indiquer des performances assez proches de celles d'ASCAT pour des vents modérés, et une très forte amélioration pour les vents extrêmes $(r<R 64)$ dans le cas des cyclones majeurs (catégorie $\geq 3$ ). Pour les événements moins intenses, les vitesses semblent néanmoins surestimées pour $\mathrm{r}<\mathrm{R} 64$, ce qui pourrait s'expliquer comme indiqué précédemment par une résolution insuffisante des données CYGNSS près du mur de l'oeil où les gradients de vitesse sont forts (et qui correspond à un rayon proche de R64 pour les cyclones les plus faibles, comme ceux de catégorie 1 et 2 en particulier).
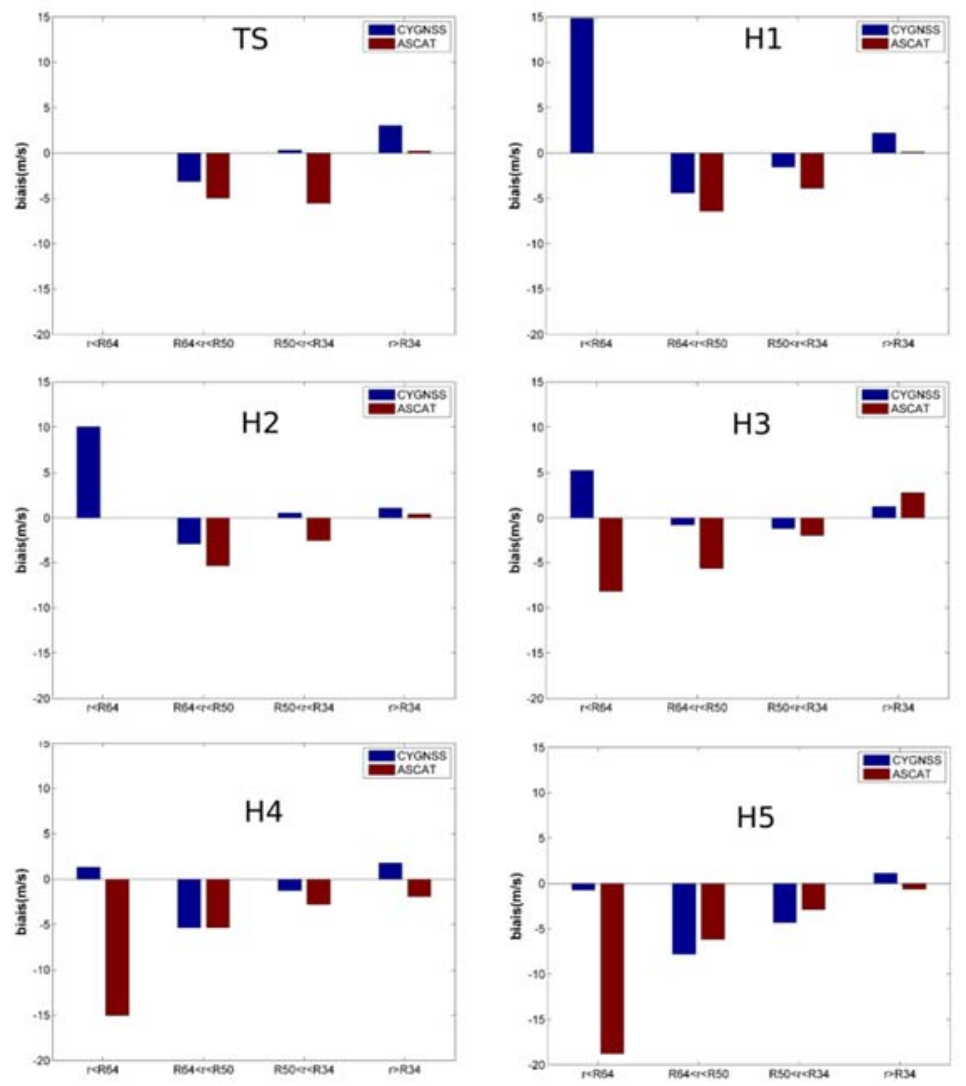

Figure 1. Biais entre les vitesses estimées par télédétection et celles déduites des formules paramétriques (des valeurs positives indiquent que les 1ères sont supérieures aux secondes). Les résultats sont présentés en fonction de l'intensité de l'événement (TS: tempête tropicale, H1-2-3-4-5:cyclone de catégorie 1-2-3-4-5), et de la distance $r$ au centre du cyclone. R34, R50 et R64 sont les rayons observés à 34, 50, et 64 næuds. 


\section{Thème 1 - Hydrodynamique côtière}

\subsection{Performance des modèles paramétriques étudiés}

Les résultats précédents suggèrent que les données ASCAT et CYGNSS pourraient constituer de bons estimateurs de la vitesse en surface pour $r>$ R34 et $r<$ R34 respectivement, à l'exception des vents les plus extrêmes $(r<R 64)$ pour les cyclones de catégorie 1 et 2 . En partant de cette hypothèse, on calcule les biais donnés par chaque formule empirique en fonction du rayon, de l'intensité de l'événement considéré, et des informations dont on dispose sur la structure des vents (R34 ou R64 uniquement, ou alors ensemble des rayons à 34,50 , et 64 nœuds). Les résultats sont représentés sur la figure 2. Ils suggèrent notamment:

a) que la performance des modèles empiriques dépend beaucoup des informations dont on dispose sur les rayons de vent à 34,50 , et 64 nœuds.

b) que parmi les modèles testés, seul E11 donne des résultats satisfaisants près de l'oeil du cyclone, notamment pour les événements les plus intenses.

c) que les modèles H80, E04, et W06 donnent de meilleurs résultats en revanche sur la partie externe des cyclones, à condition qu'ils soient bien calibrés pour des vents modérés (informations sur R34 par exemple).

Ces résultats sont cohérents avec ceux présentés dans les travaux publiés (ex: WILLOUGHBY et al., 2006, EMANUEL \& ROTUNNO, 2011). Ils montrent aussi une réduction significative des biais autour des rayons à 64 nœuds et 34 nœuds, selon que l'on contraint les modèles empiriques avec R64 ou R34 respectivement. Ceci indique que les données de télédétection sont cohérentes avec chaque formule empirique considérée indépendamment (et non pas seulement la moyenne, comme dans la section précédente). Ces résultats confortent donc l'hypothèse que les données ASCAT et/ou CYGNSS peuvent servir d'estimateurs pour les vents cycloniques dans la plupart des cas de figure.

\subsection{Comparaison avec des données-in situ}

Un modèle couplé courants-vagues a été implémenté pour tenter de reproduire les états de mer associés à l'ouragan Maria, qui a touché les Antilles Françaises en septembre 2017. Plusieurs formules empiriques ont été testées pour le forçage du modèle avec des champs de vent. Les résultats, que le lecteur intéressé pourra trouver dans KRIEN et al. (2018), confirment une fois de plus ceux de la figure 2, et donnent encore plus de poids à l'hypothèse faite précédemment sur l'utilité des données spatiales telles que CYGNSS.

\section{Conclusions}

Ce travail suggère que la télédétection spatiale permet aujourd'hui de mieux représenter les champs de vent cycloniques, et donc de choisir de manière plus objective les modèles paramétriques qui permettront de mieux évaluer les impacts de ces événements extrêmes. La capacité des modèles à représenter correctement les champs de vent 


\section{XVèmes Journées Nationales Génie Côtier - Génie Civil La Rochelle, 29 au 31 mai 2018}

dépend par ailleurs beaucoup des informations dont on dispose sur la structure des cyclones (rayons à 34, 50, et 64 nœuds par exemple).

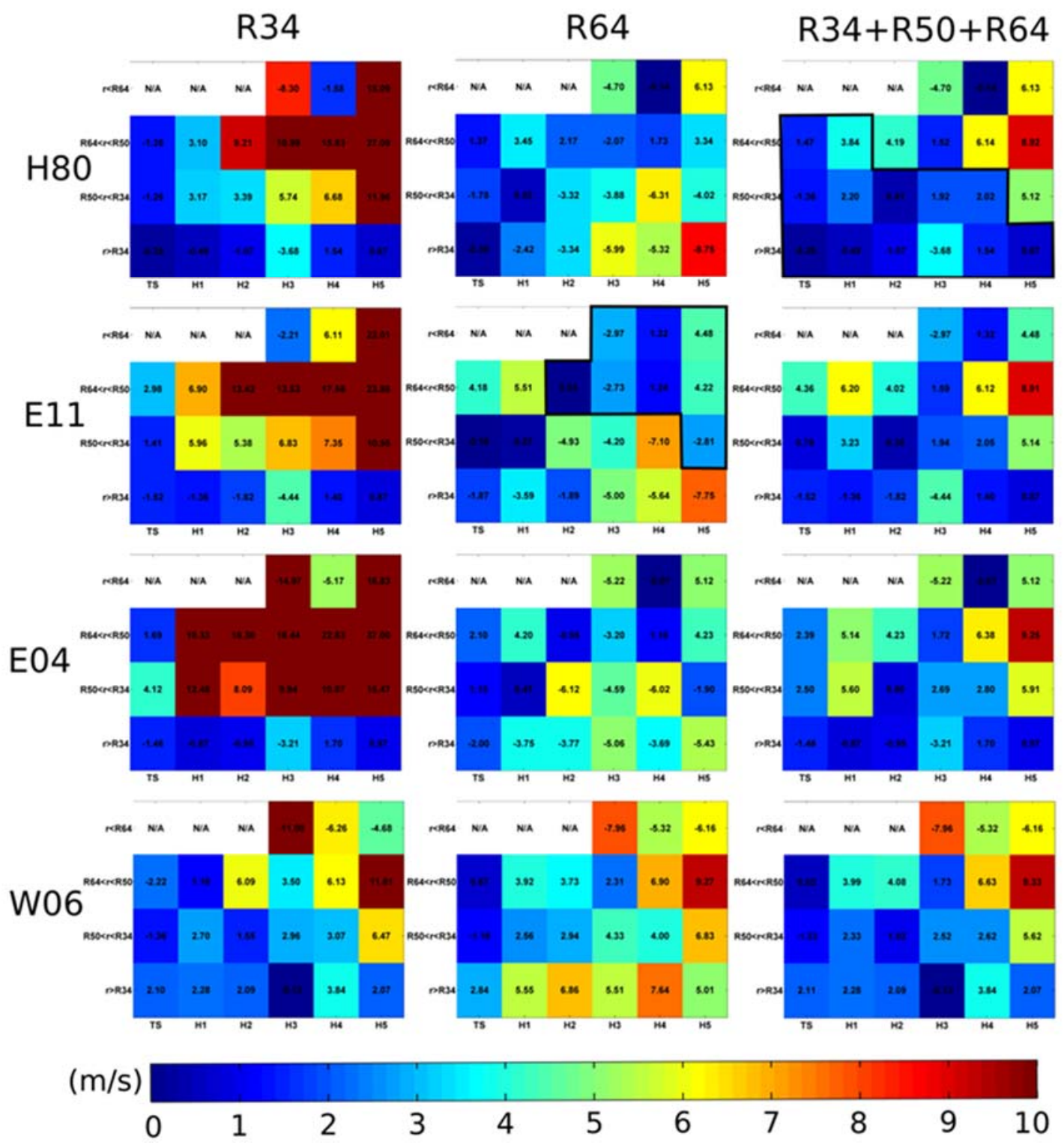

Figure 2. Diagrammes représentant les biais sur les vitesses en fonction de la formule paramétrique considérée, de l'intensité de l'événement(TS: tempête tropicale, H1-2-3-4-

5:cyclone de catégorie 1-2-3-4-5), de la distance r au centre du cyclone, et des informations dont on dispose (R34, R50 et R64 sont les rayons observés par le NHC à

34, 50, et 64 nœuds). La barre de couleur indique la valeur absolue du biais. Les contours noirs suggèrent un choix de modèle susceptible de donner des bons résultats:

E11 près du centre, et H80 plus loin (modèle testé dans KRIEN et al., 2018). 


\section{Thème 1 - Hydrodynamique côtière}

\section{Références bibliographiques}

EMANUEL K. (2004). Tropical cyclone energetics and structure, in Atmospheric Turbulence and Mesoscale Meteorology, edited by E. Fedorovich, R. Rotunno, and B. Stevens, pp 165-192, Cambridge Univ. Press, Cambridge, U.K. https://doi.org/10.1017/CBO9780511735035.010

EMANUEL K., ROTUNNO R. (2011). Self-stratification of tropical cyclone outflow, Part I: Implications for storm structure, J. Atmos. Sci., Vol. 68, pp 2236-2249. https://doi.org/10.1175/JAS-D-10-05024.1

FIGA-SALDANA J., WILSON J.J.W., ATTEMA E., GELSTHORPE R., DRINKWATER M.R., STOFFELEN A. (2002). The advanced scatterometer (ASCAT) on the meteorological operational (MetOp) platform: A follow on for European wind scatterometers. Canadian Journal of Remote Sensing, Vol. 28, pp 404-412. https://doi.org $/ 10.5589 / \mathrm{m} 02-035$

HOLlAND G. (1980). An Analytic Model of the Wind and Pressure Profiles in Hurricanes. Monthly Weather Review, Vol. 108, pp 1212-1218. https://doi.org/10.1175/15200493(1980)108<1212:AAMOTW $>2.0$. CO; 2

HU K., CHEN Q., KIMBALL S.K. (2012). Consistency in hurricane surface wind forecasting : an improved parametric model. Nat. Hazards, Vol. 61, pp 1029-1050. https://doi.org/10.1007/s11069-011-9960-z

KRIEN Y., ARNAUD G., CECE R., KHAN J., BEL MADANI A., BERNARD D., ISLAM A.K.M.S., DURAND F., TESTUT L., PALANY P., ZAHIBO N. (2018). Improving Parametric Cyclonic Wind Fields Using Recent Satellite Remote Sensing Data. Preprints 2018, 2018030095.

LIN N., CHAVAS D. (2012). On hurricane parametric wind and applications in storm surge modeling. J. Geophys. Res. Vol. 117, D09120. https://doi.org/10.1029/2011JD017126

MORRIS M., RUF C. (2017). Determining Tropical Cyclone Surface Wind Speed Structure and Intensity with the CYGNSS Satellite Constellation. J. Appl. Meteor. Climatol. https://doi.org/10.1175/JAMC-D-16-0375.1

RUF C. S., and Coauthors. (2016). New Ocean Winds Satellite Mission to Probe Hurricanes and Tropical Convection. Bull. Amer. Meteor. Soc., Vol. 97, pp 385-395. https://doi.org/10.1175/BAMS-D-14-00218.1

RUF, C. S., GLEASON, S., MCKAGUE, D.S. (2018). Assessment of CYGNSS Wind Speed Retrieval Uncertainty. IEEE J. 557 Sel. Topics Appl. Earth Obs. Remote Sens.

WILLOUGHBY H.E., RAHN M.E. (2004). Parametric representation of the primary hurricane vortex. Part I: Observations and evaluation of the Holland (1980) model. Mon. Wea. Rev., Vol. 132, pp 3033-3048. https://doi.org/10.1175/MWR2831.1

WILLOUGHBY H.E., RAHN M.E., DARLING R.W.R. (2006). Parametric representation of the primary hurricane vortex. Part II: A new family of sectionally continuous profiles. Mon. Weath. Rev., Vol. 134, pp 1102-1120. https://doi.org/10.1175/MWR3106.1 